\title{
Using a GIS to Assessment the Load-Carrying Capacity of Soil Case of Berhoum Area, Hodna Basin, (Eastern Algeria)
}

\author{
Amar Guettouche, Farid Kaoua \\ Faculty of Civil Engineering, University of Sciences and Technology Houari Boumediene, Algiers, Algeria \\ Email: guettouche.amar@gmail.com,ammar.bahri73@yahoo.fr
}

Received July 2, 2013; revised August 9, 2013; accepted October 2, 2013

Copyright (C) 2013 Amar Guettouche, Farid Kaoua. This is an open access article distributed under the Creative Commons Attribution License, which permits unrestricted use, distribution, and reproduction in any medium, provided the original work is properly cited.

\begin{abstract}
The concept of load-carrying capacity of the soil can be evaluated by two main components: permissible stress and permissible depth; and therefore, running it begins its assessment that allows an outline of exploitation. Nevertheless, the assessment of the load-carrying capacity made the object of several works of research and many models, based on the multi-criteria analysis, have been established. This work examines the contribution of GIS approach to assessment load-carrying capacity of the soil. This one has been finished in two practicums: 1) Assessment of the capacity of soil by a multi-criteria approach, using the Weighted Sum Model (WSM); 2) It brought to use the GIS approach to evaluate and spatialize degree of soil bearing stresses resulting from the buildings, as well as load distribution. The method has been applied to the Berhoum area of Hodna Basin, in eastern Algeria, where each is characterized by its various natural properties and density of equipment. Final results are better in the classification of the degree of load-carrying capacity possible in each site. This results in allowing exploiters to program their optimal designs for the rational management of the area.
\end{abstract}

Keywords: Load-Carrying Capacity; WSM; GIS; Berhoum; Hodna Basin's; Algeria

\section{Introduction}

Some Algerian middle areas are witnessing difficulties in construction caused by problems related to soil, especially the instability of foundations. This is due to the weakness of knowledge of soil characteristics like: its ability to bear the load of buildings and geological composition. Conversely, these areas are characterized by population density and speed of urban growth.

The danger is caused by these problems which are annoyed and still disturbing the population life progress. However, it leads to negative effects on working life in these areas and thus, evacuating them. So, the preventive methods remain the best way to overcome these problems or reduce them.

One of the means that allows the adoption of effective preventive methods is to understand the phenomenon and predict the places where it occurs. And this is what matters in our research, which aims to assess the load-carrying capacity of soil mathematically using the intersection approach relying on the calibration analysis and to calibrate it according to field measurements.

\section{The Study Area}

The area that has been selected as a study area is Berhoum, (previously Souk Ouled Nedjaa), in El Hodna Basin, Eastern Algeria which is characterized by its various natural properties and density of equipment in it. It is located at a distance of $300 \mathrm{~km}$ south of Algiers and $50 \mathrm{~km}$ to the east of the city of M'sila (Figure 1).

\section{Methodology}

It is known that the geotechnical mapping, including Multi evaluation of load-carrying capacity of the soil, was the subject of a lot of research scientific work, including: [1-7].

Many relationships and mathematical models that have been developed either partial; depend on the standard one or several criteria mostly "geographical" and not "Geotechnical". 


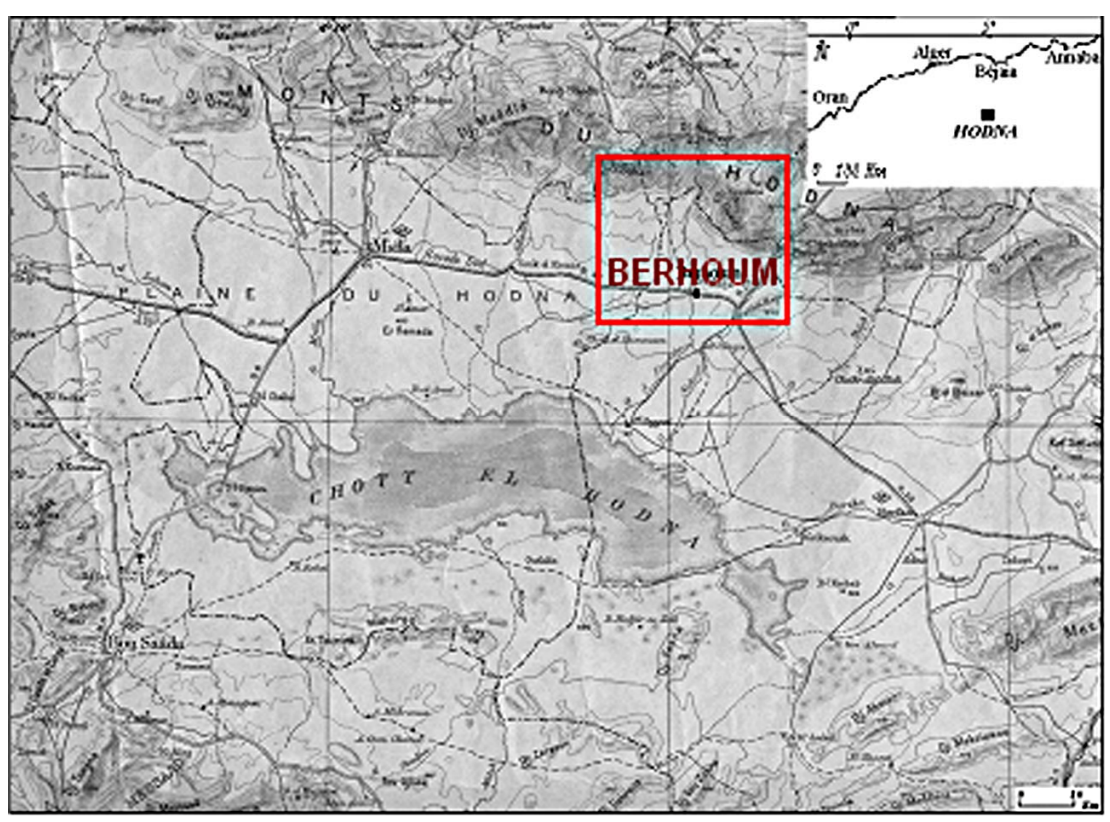

Figure 1. Location of the study area.

On the other hand, the endurance or the load-carrying capacity of soil is known as the maximum pressure that can be borne by the soil before the collapse happens in the construction ground $[8,9]$.

Based on this definition, the load-carrying capacity of soil is associated with two basic components: the soil allowable pressure and the depth at which the pressure is applied. And by identifying the factors that control the load-carrying capacity of the construction soil, a mathematical model has been adopted. We review it as follows.

\subsection{Hypotheses}

We pose, in this occasion, two basic assumptions:

The load-carrying capacity of soil is a phenomenon that can be evaluated by geotechnical factors related to soil.

The possibility of the phenomenon occurrence is expressed by the total weighted factors leading to it.

\subsection{Modeling Load-Carrying Capacity}

Load-carrying capacity of soil $(P)$ is the sum of the determinants of the phenomenon, but each factor has its weight (WSM) and it is expressed in the following relationship:

$$
P=\sum_{l}^{n} w_{i} \cdot C_{i}
$$

where:

$w_{i}$ : gravity or weight specified for each worker.

$C_{i}$ : determinants.

For the load-carrying capacity of soil; the first stage of modeling begins by choosing the geotechnical criteria that intervene or help in the process of its occurrence, its spread, its intensity, or all combined. These criteria are in fact interrelated and therefore their intersection does the phenomenon. Consequently, the load-carrying capacity of soil can be found depending on WSM for both stress and depth. This scientific explanation can be expressed as follows:

$$
P=0.6 \sigma_{\text {perm }}+0.4 H_{\text {perm }}
$$

where:

$P$ : the load-carrying capacity of soil.

$\sigma_{\text {perm }}$ : permissible stress of the soil.

$H_{\text {perm }}$ : the permissible depth of the soil (depth at which applied stress).

In this method, the weight values, and classification is based on expert opinion.

\subsubsection{Permissible Stress of the Soil}

The permissible stress is one of the main factors that govern the stability of the foundations and it has been prepared $\left(\sigma_{\text {perm }}\right)$ taking into account the highest recorded stresses.

Taking into account the lithology map, and geotechnical surveys available, the permissible stresses for each lithologic facies collected then the permissible stress of the entire region is determined.

The permissible stress values are divided into four main categories (Table 1).

\subsubsection{Permissible Depth of Soil}

The permissible depth $\left(H_{\text {perm }}\right)$ has been prepared accounting the largest registered depths which agreed for 
Table 1. Classification and grade of the criteria specifying permissible stress.

\begin{tabular}{ccc}
\hline Permissible stress & Grade & Classification \\
\hline$\sigma_{\text {perm }}<1$ bar & 1 & Low \\
1 bar $\leq \sigma_{\text {perm }}<2$ bars & 2 & Medium \\
2 bars $\leq \sigma_{\text {perm }}<3$ bars & 3 & Strong \\
$\sigma_{\text {perm }} \geq 3$ bars & 4 & Very strong \\
\hline
\end{tabular}

the recorded permissible stresses. And depending on the lithology map and the available geotechnical surveys; the permissible depths for each lithological face have been collected then they have been distributed in the entire region.

The permissible depth values have been divided into four main categories (Table 2).

\subsection{Intersection between the Standards and the Completion of Tables}

In order to be able to use the proposed model to assess the load-carrying capacity of soil, we first identify the factors controlling the phenomenon and classify them then digitize them by the degree of impact of each factor. Relying on quantitative data, to assess the load-carrying capacity of soil, we used a Weight Somme Model (WSM) where the rank of weights and values are in Table 3.

Intersection matrix is given as Table 4.

Intersection between stress and depth permissible they give the strapless soil foundations as shown in Table 4.

\section{Spatialization by GIS Approach: Application in the Study Area}

The approach we have adopted for the spatial distribution map preparation to assess the load-carrying capacity of soil is based on the geographic information systems in which we used two types of data:

-The first is related to the lithological map of the area (rocks' map),

-And the second is related to data of the field survey and geotechnical drilling.

These data obtained from the rocks' map or from the field survey and geotechnical drilling, have been implemented in GIS program (MapInfo 8) for the primary maps drawing.

After the building of a database of the geographic reference, organizing and structuring it by the use of programs, a map of the load-carrying capacity of soil that shows the best areas for building.

\subsection{Lithological Map of Study Area}

The rocky map of the area is derived from the geological map "Souk Ouled Nedjaa" (map No. 169, scale 1:50.000), prepared by the R. Guiraud [10]. It has been prepared
Table 2. Classification and grade of the criteria specifying permissible depth.

\begin{tabular}{ccc}
\hline Permissible depth & Grade & Classification \\
\hline$H_{\text {perm }} \geq 2 \mathrm{~m}$ & 1 & Low \\
$1.5 \mathrm{~m} \leq H_{\text {perm }}<2 \mathrm{~m}$ & 2 & Medium \\
$1 \mathrm{~m} \leq H_{\text {perm }}<1.5 \mathrm{~m}$ & 3 & Strong \\
$H_{\text {perm }}<1 \mathrm{~m}$ & 4 & Very strong \\
\hline
\end{tabular}

Table 3. Order of weights and values of the criteria.

\begin{tabular}{cccc}
\hline Criteria & Weights & Classification & Values \\
\hline \multirow{3}{*}{$\sigma_{\text {perm }}$} & \multirow{3}{*}{0.6} & Low & 0.6 \\
& & Medium & 1.2 \\
& & Strong & 1.8 \\
& & Very strong & 2.4 \\
$H_{\text {perm }}$ & \multirow{3}{*}{0.4} & Low & 0.4 \\
& & Medium & 0.8 \\
& & Strong & 1.2 \\
& & Very strong & 1.6 \\
\hline
\end{tabular}

Table 4. Degree of the load-carrying capacity of soil.

\begin{tabular}{ccccc}
\hline$\sigma_{\text {perm }} H_{\text {perm }}$ & 0.6 & 1.2 & 1.8 & 2.4 \\
\hline 0.4 & 1 & 1.6 & 2.2 & 2.8 \\
0.8 & 1.4 & 2 & 2.6 & 3.2 \\
1.2 & 1.8 & 2.4 & 3 & 3.6 \\
1.6 & 2.2 & 2.8 & 3.4 & 4 \\
\hline
\end{tabular}

Colours reflect the degree of load-carrying capacity $(\mathrm{P})$.

after the geological map numeration, then building the rocky facets' database. After the topical analysis, the following major rock, units has been identified (Figure 2).

\subsection{Field Survey Data and Geotechnical Borehole}

To complete the database we have adopted central values of all the criteria selected for this study. Data used in this study were collected from 49 study geotechnical surveys, well field investigation based on direct observations, as well as recordings and field sampling (GPS, images, etc.).

Geotechnical studies prepared soil laboratories: LHCCM'sila, LCTP-M'sila, LIEG-M'sila, AICH.GEOSOL-Setif, EAST.LCTP-Setif, and LBEMA-Bordj Bou Arreridj.

These data were consolidated and exploit digitization units have been analysed and evaluated these data for my map permissible stress $\left(\sigma_{\text {perm }}\right)$ and permissible depth $\left(H_{\text {perm }}\right)$ (Figures 3 and 4).

\subsection{Identification and Classification of Load-Carrying Capacity}

Depending on the relationship (1) that have been entered into of GIS program guide, strapless been assessing of capacity of the soil $(P)$ according to of proposed classifycation previously, She of output of map shown in Figure 5. 


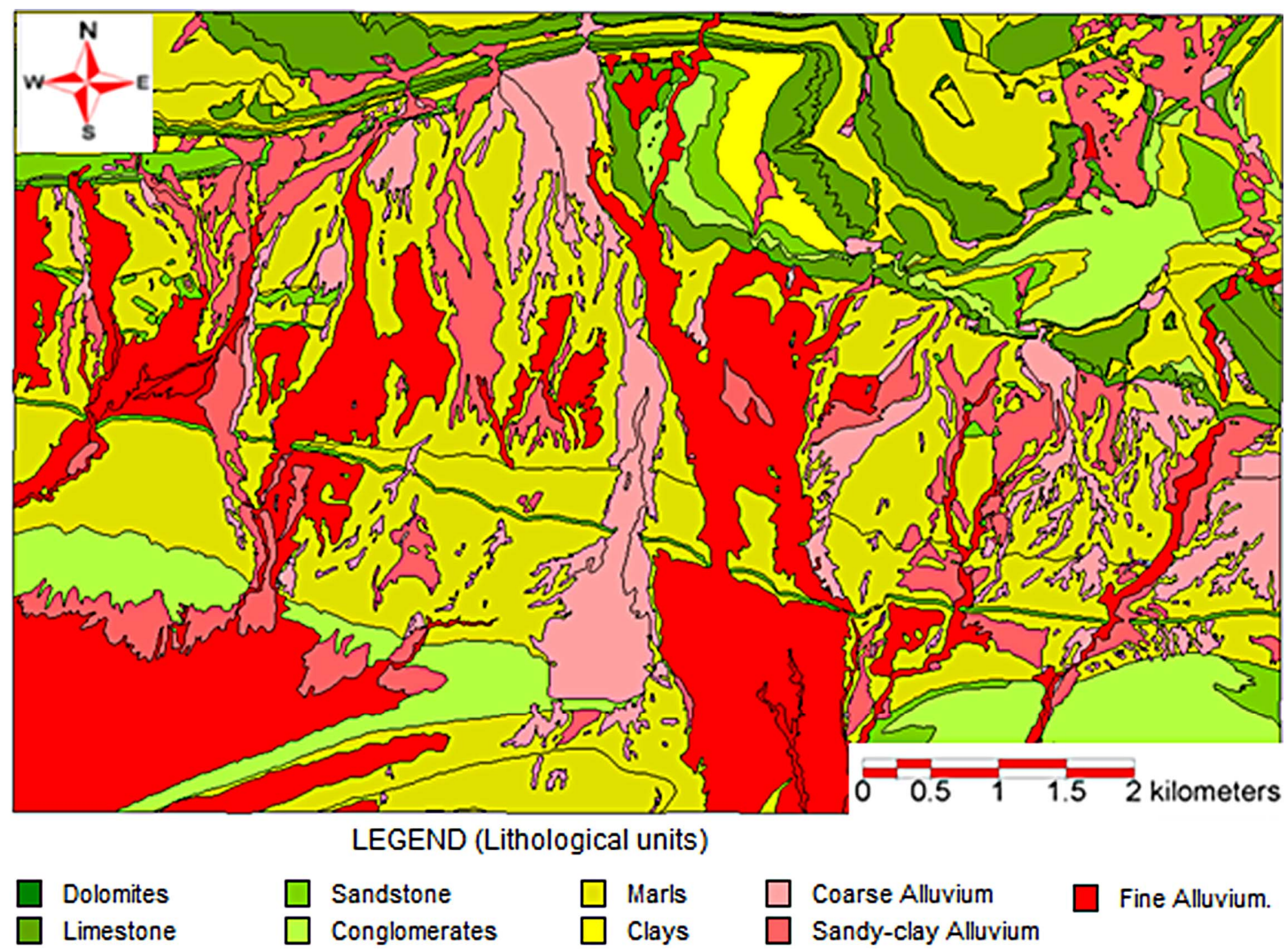

Figure 2. The main rock units of the study area.

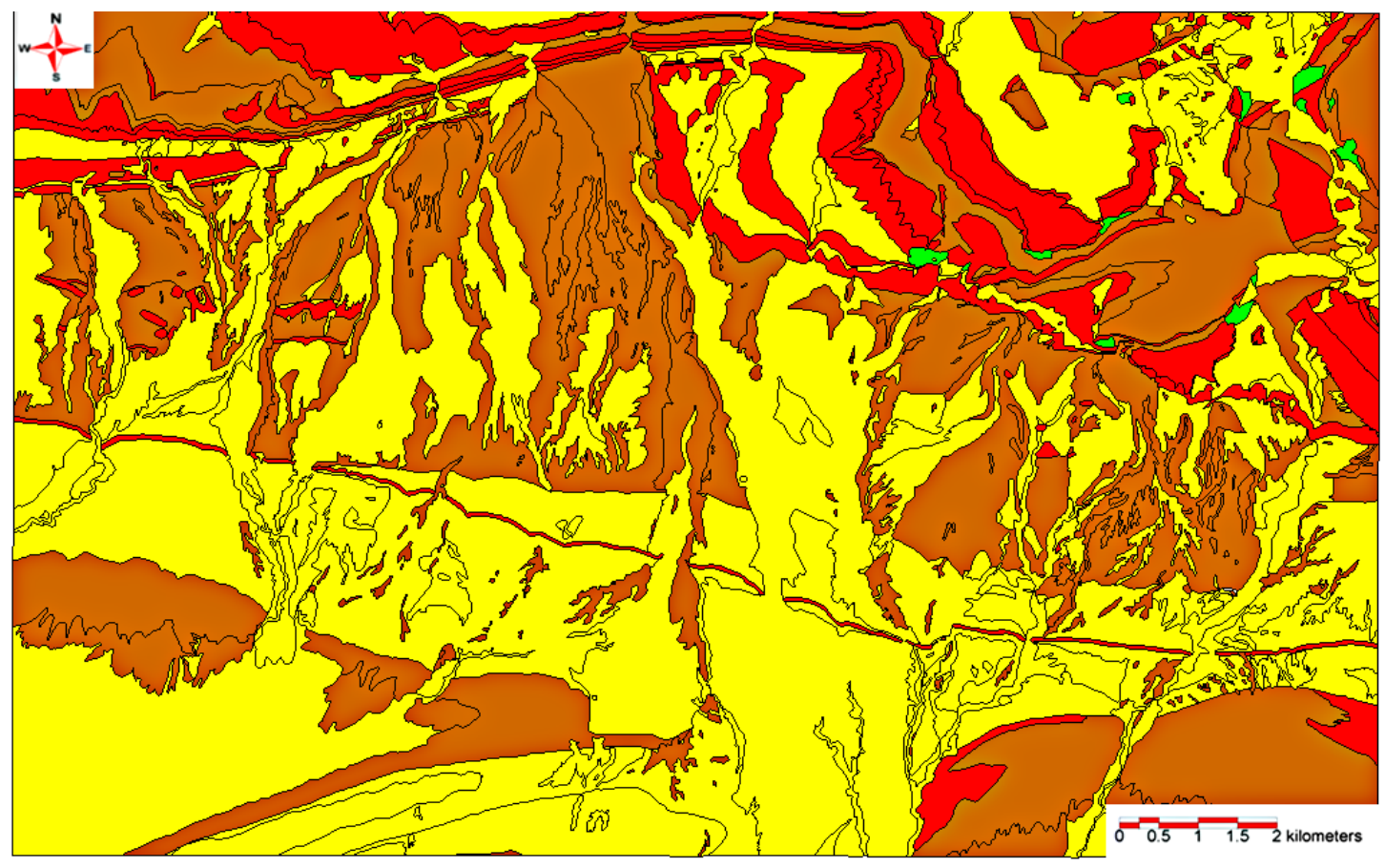

LGENDE :

Permissible Stress Degre

Low $\square$ Medium

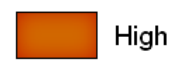

High

Very High

Figure 3. The permissible stress map of study area. 


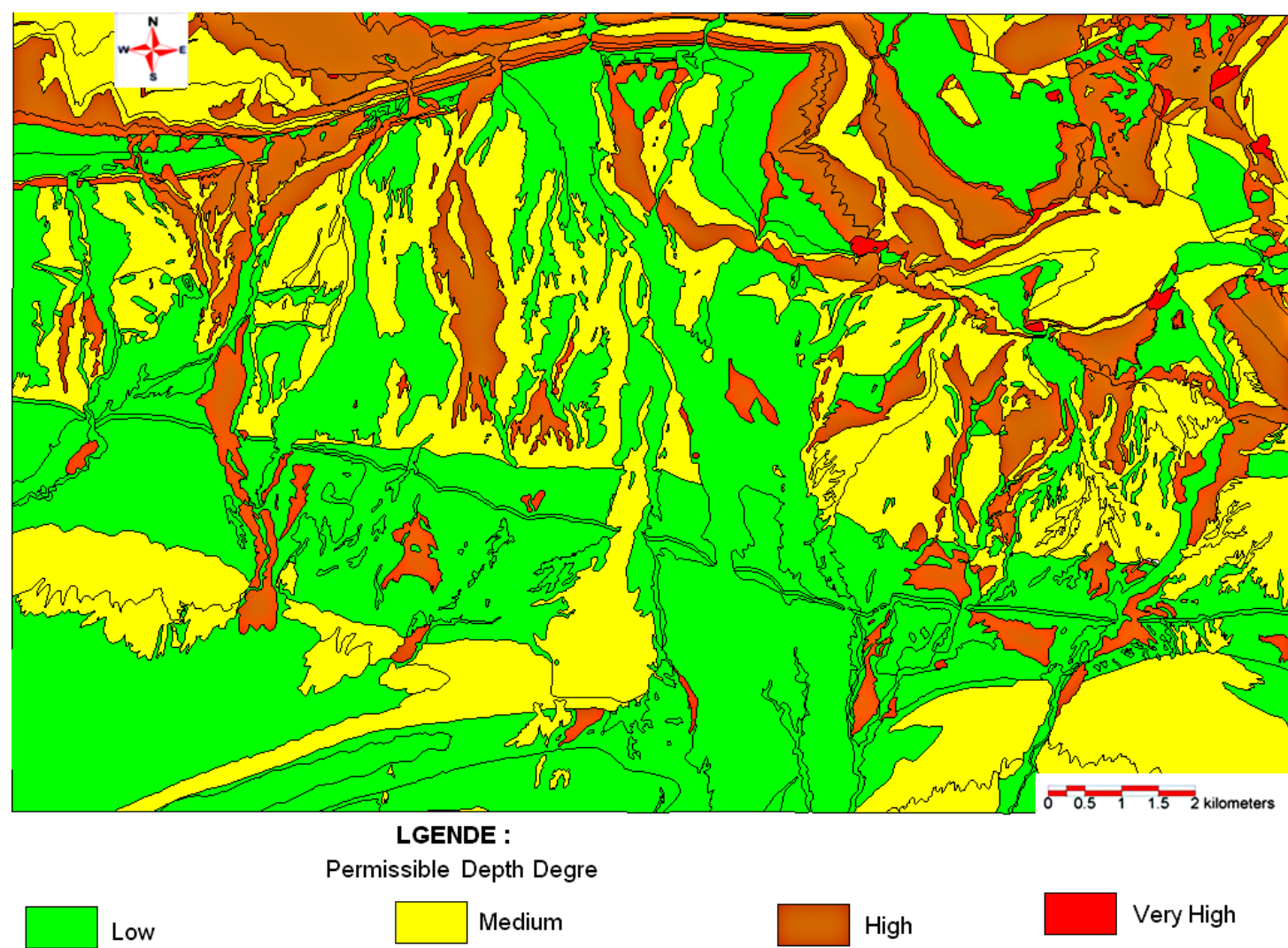

Figure 4. The permissible depth map of study area.

\section{Discussion}

The use of geographic information systems (GIS) with the adoption of (WSM) model allowed and allows fast the preparation of the geotechnical maps, the load carrying capacity of soil map in particular; which requires the combination of great amount of spatial data with many different geographic data, experts data and decision-makers details.

The digital technology has been used in drawing the rocky map of the study area that formed the basis which we have adopted in the completion of the main rock units of that region.

Field survey data and geotechnical drilling allowed the data collection to get the permissible stress $\left(\sigma_{\text {perm }}\right)$ map and the permissible depth $\left(H_{\text {perm }}\right)$ maps (Figures 2 and $\left.\mathbf{3}\right)$. Thees data have been used to calculate the field' loadcarrying capacity of the soil (Figure 4) by the relationship (1).

Based on the concept of the intersection, the proposed model is simple, and easily used by the geotechnical engineer or otherwise. Thread model is very interesting as it is being built on a small number of geotechnical charcteristics according to experts' judgment. Similarly, this model can be easily applied to other similar regions in terms of the environmental landscape and climate. This model is not static; however, it can be changed some- times by giving a great attention to one factor and decrease it to another.

Because of the obvious advantages of this method, the load-carrying capacity of the soil map of the study area (Figure 4) has been selected to be the final map for the study.

\section{Conclusions}

This study demonstrates the importance of the geographic information systems (GIS) techniques which use with a Weighted Somme model (WSM) to prepare the load carrying capacity of the soil that is important in determining the efficiency of residential areas for the completion of construction projects.

The significant advantages of using these techniques can be summarized in: the low cost, the easily use data, quickly update data and the ability to produce new scenarios.

In this study, the permissible depth and the permissible stress maps have been prepared for the selected area. Weights values, the analysis and the classification have been assigned by (WSM) model. As a result, the study area has been divided into four different areas according to the foundations efficiency: 1) areas with a weak load carrying capacity of soil; 2) areas with a medium load carrying capacity of soil; 3 ) areas with a strong load car- 


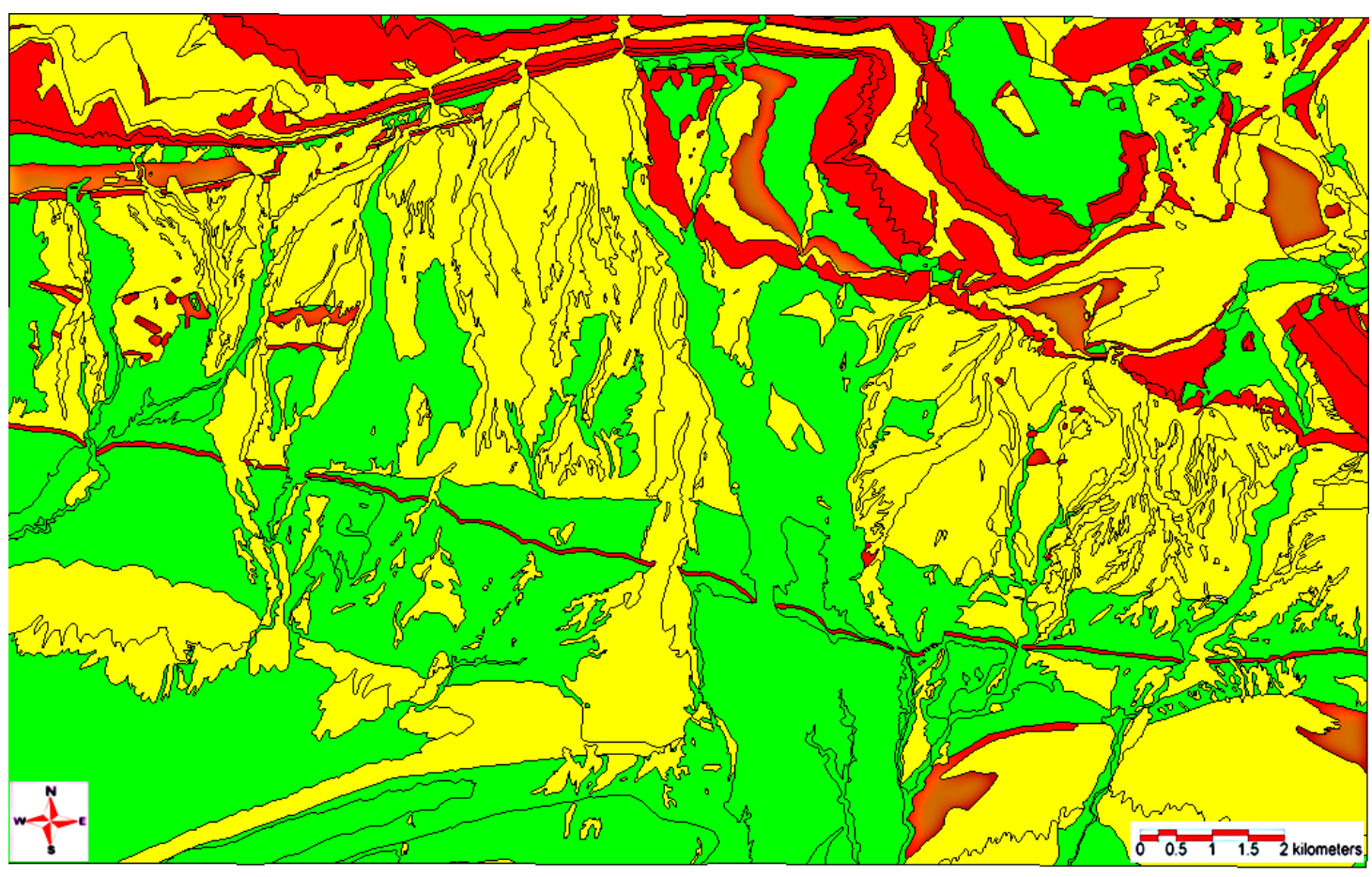

LGENDE :

Load-carrying capacity

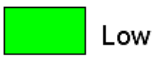

Medium

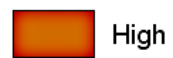

Very High

Figure 5. Load-carrying capacity of soil in study area.

rying capacity of soil; and 4) areas with very strong load carrying capacity of soil. According to this map, most of the study area was identified suitable for construction.

The methodology used for the selected area in this study can be applied to other places and other procedures for the selection of the site and be used to rebuild the necessary standards appropriately.

\section{REFERENCES}

[1] C. Ayday, M. Altan, H. A. Nefeslioğlu, A. Canigür, S. Yerel and M. Tün, "Preparation of Engineering Geological Map of Eskişehir Urban Area," Research Institute of Satellite and Space Sciences, Anadolu University (in Turkish), 2001.

[2] S. J. Carver, "Integrating Multicriteria Evaluation with Geographical Information Systems," International Journal of Geographical Information Systems, Vol. 5, No. 3, 1991, pp. 321-339.

http://dx.doi.org/10.1080/02693799108927858

[3] J. T. Diamond and J. R. Wright, "Design of an Integrated Spatial Information System for Multiobjective Land Use Planning," Environment and Planning B, Vol. 15, No. 2, 1988 , pp. $205-214$.

http://dx.doi.org/10.1068/b150205
[4] J. R. Eastman, W. Jin, P. A. K. Kyem and J. Toledano, "Raster Procedures for Multicriteria/Multiobjective Decisions," Photogrammetric Engineering and Remote Sensing, Vol. 61, No. 5, 1995, pp. 539-547.

[5] P. Jankowski, "Integrating Geographical Information Systems and Multiple Criteria Decision Making Methods," International Journal of Geographical Information Systems, Vol. 9, No. 3, 1995, pp. 251-273. http://dx.doi.org/10.1080/02693799508902036

[6] C. P. Keller, "Decision Making Using Multiple Criteria," NCGIA Core Curriculum, Unit 57, Santa Barbara, National Center for Geographic Information and Analysis, 1996. http://www.geog.ubc.ca/courses/klink/

[7] J. Malczewski, "GIS and Multicriteria Decision Analysis," John Willey and Sons Inc., Hoboken, 1999, 392 p.

[8] K. Meftah, “Cours Mécanique des Sols,” 2008, pp. 70-72. http://pf-mh.uvt.rnu.tn/

[9] G. Philipponnat and B. Hubert, "Fondations et Ouvrages en terre, Troisième Tirage," Eyrolles, 2002, p. 548.

[10] R. Guiraud, "Notice Explicative de la Carte Géologique au 1/50.000è de Souk Ouled Nedja," Publication du Service Géologique de l'Algérie, Pl. Hors-texte, 1971, 35 p. 